\title{
Le dépistage biologique d'une conduite sous influence
}

\section{Toxicological detection of driving under the influence}

\section{Alain G. VERSTRAETE(1), Nele SAMYN( ${ }^{(2)}$}

(1) Laboratoire de Biologie Clinique, Hôpital Universitaire De Pintelaan 185, B-9000 GENT - BELGIQUE

Tél : +3292403407- Fax : +3292404985

(2) Institut National de Criminalistique et Criminologie, section toxicologie, Chaussée de Vilvorde 98-100,

B-1120 BRUXELLES - BELGIQUE

(Reçu le 14 avril 2003 ; accepté le 30 avril 2003)

\section{$R E ́ S U M E ́$}

Au cours des 5 à 7 dernières années, beaucoup de progrès ont été faits dans le domaine des méthodes de détection des drogues dans le cadre de la conduite automobile: tests de terrain, seuils de détection optimaux et méthodes de laboratoire. La nécessité de disposer d'un test rapide fiable est bien établie. Les tests rapides urinaires sont assez fiables, mais ils posent le problème de la collecte de l'urine sur le terrain. Le développement des tests sur la salive a été plus lent que prévu, et les problèmes majeurs à résoudre sont la sensibilité pour le tétrahydrocannabinol et l'obtention d'un échantillon satisfaisant (volume et viscosité). Les seuils SAMHSA (Substance Abuse and Mental Health Service Administration) qui ont été proposés pour la salive sont également applicables pour les cas de conduite sous influence de drogues. Il y a peu de nouveautés dans les tests rapides sur la sueur. Les seuils analytiques des drogues dans le sang varient dans les différents pays européens et l'Allemagne les a récemment revus à la baisse. L'analyse de drogues dans le sang est maintenant devenue une procédure de routine dans beaucoup de laboratoires, qui utilisent une méthode de chromatographie en phase gazeuse (parfois aussi en phase liquide) couplée à la spectrométrie de masse, éventuellement précédée d'un dépistage immunologique.

\section{MOTS-CLÉS}

Conduite automobile, stupéfiants, alcool, tests de terrain, seuils, législation.

\section{SUMMARY}

Much progress has been made in the last 5 to 7 years in the field of analytical detection methods for driving under the influence of drugs: roadside tests, optimal cut-offs and laboratory methods. The need for a roadside drug test is now well established. Urine onsite tests work well but the problem of obtaining a urine specimen at the roadside remains. The development of oral fluid tests has been slower than expected, with the sensitivity for tétrahydrocannabinol and adequate (volume and viscosity) sampling remaining the major problems. The proposed SAMHSA (Substance Abuse and Mental Health Service Administration) cut-offs for oral fluid seem also applicable for driving under the influence of drugs. There has been little development of sweat tests. The analytical cut-offs used differ in European countries and Germany has recently decreased them. Analysis of drugs in blood has now become routine in many labs, either by $G C$ $M S$, but also by LC-MS, sometimes preceded by an immunoassay screening.

\section{KEY-WORDS}

Impaired driving, illicit drugs, alcohol, roadside tests, cutoffs, legislation. 


\section{Introduction}

La détection d'une conduite sous influence d'alcool a connu une évolution progressive. Il y a cinquante ans, celle-ci n'était pas réprimée, au contraire, le fait d'être sous l'emprise de l'alcool était considéré comme une circonstance atténuante. Plus tard, ces conducteurs ont été reconnus sous influence par des tests comportementaux (Standard field sobriety test des américains). Ensuite, le législateur, se basant entre autres sur les résultats de l'étude de Grand Rapids (1), a introduit des seuils légaux, qui ont été progressivement revus à la baisse. Par exemple, en Belgique, le seuil était de $1,5 \mathrm{~g} / \mathrm{L}$ en 1958 , puis il a été progressivement abaissé à $0,8 \mathrm{~g} / \mathrm{L}$ en 1973 et $0,5 \mathrm{~g} / \mathrm{L}$ en 1994 . D'autres pays ont été plus clairvoyants: la Norvège a introduit un seuil légal de $0,5 \mathrm{~g} / \mathrm{L}$ en 1936 !

On remarque la même évolution concernant la conduite sous influence de drogue. Beaucoup de pays avaient une législation non spécifique, et la conduite sous influence de drogue faisait référence à un article général qui interdisait la conduite en état d'ivresse, quelle qu'en soit la cause. Comme il était très difficile de prouver que le conducteur était sous influence, cet article était très peu appliqué. En 1998, l'Allemagne, bientôt suivie par la Belgique et la Suède, a introduit une législation analytique ou "per se" : Le conducteur est considéré comme étant sous influence si une drogue ou un métabolite spécifique est présent dans le sang.

En parallèle avec l'évolution de la législation concernant l'alcool, on a cherché à développer des tests de terrain pour la détection d'une conduite sous influence. Comme il n'était pas possible de faire l'analyse dans le sang, on a cherché des matrices alternatives, l'air expiré pour l'alcool. Grace à l'évolution technologique les appareils actuels sont très fiables, et la plupart des interférences sont corrigées.

Une évolution analogue s'observe pour les drogues. La police recherche des tests de terrain et ici aussi, comme il n'est pas aisé de disposer de sang ou d'un échantillon urinaire au bord de la route, on explore les possibilités des matrices alternatives: la salive et la sueur. La technique analytique pour le dépistage est l'immunoessai, dont le manque de spécificité est bien connu.

\section{Dépistage rapide d'une conduite sous influence}

\section{Est-il nécessaire de disposer d'un test rapide pour la détection des drogues?}

A l'heure actuelle, iI existe un consensus scientifique selon lequel seule une analyse des drogues dans le sang peut constituer une preuve d'une conduite sous influence. On peut donc se poser la question de savoir si d'autres tests sont nécessaires, car si le conducteur est supposé être sous influence de drogues, on peut faire une prise de sang, et on n'a pas besoin d'autres tests. L'expérience a montré que ce raisonnement est trop simpliste. Dès 1999, Krüger écrivait qu'il y avait un besoin de développer un test de terrain fiable, rapide et bon marché pour la détection des drogues les plus fréquentes (2).

Etant donné que le prélèvement est invasif, qu'il doit toujours être effectué par du personnel médical et que l'analyse de l'échantillon prend du temps et est relativement coûteuse, un test rapide d'orientation sur le terrain est utile, par analogie avec l'éthylotest. Ce test est nécessaire afin de pouvoir prendre des mesures immédiates, notamment une interdiction de conduire pour une durée déterminée, ou la prise de sang pour analyse ultérieure en laboratoire pour confirmer ce résultat. Un test de terrain peut être utile sous les deux types de législation: capacité de conduite diminuée ("impairment") ou analytique ("per se").

Pendant le projet ROSITA (Roadside Testing Assessment), une enquête dans 16 pays a permis de connaître les besoins des forces de police. Les besoins et contraintes different selon les pays, mais il existe un désir commun de disposer d'un test multiparamétrique permettant de fournir un résultat non ambigu en 5 minutes pour la détection du cannabis, des amphétamines, de la cocaïne, des opiacés et des benzodiazépines. La salive est considérée comme la meilleure matrice pour effectuer les tests ; la sueur est considérée comme acceptable et l'analyse des urines est jugée inacceptable dans certains pays (3).

Le projet ROSITA a aussi permis de confirmer le besoin d'un test rapide. Les tests rapides facilitent l'application de la loi et permettent d'économiser du temps (en évitant de devoir emmener le conducteur suspect au poste de police) et de l'argent (en limitant l'analyse de sang plus coûteuse aux cas où les chances de positivité seront plus grandes). L'utilisation d'un test rapide augmente la confiance du policier (car elle permet de confirmer sa suspicion initiale) et l'encourage à verbaliser les cas de conduite sous influence de drogue. L'étude a également démontré que les officiers de police n'avaient pas d'objections à prélever des échantillons et à utiliser les tests. Les conducteurs sont souvent impressionnés par le résultat du test, et si au départ ils nient avoir pris de la drogue, un résultat positif va souvent les faire avouer. L'utilisation de tests de terrain et la publicité qui est faite à ce sujet ont un effet de dissuasion, parce que le risque subjectif d'être pris aug- 
mente (4).

En Belgique, un test urinaire rapide est inclus dans la procédure légale (5). Dans d'autres pays comme l'Allemagne, ces tests sont utilisés couramment (6).

\section{Choix de l'échantillon biologique pour le test rapide}

Le choix du liquide biologique (urines, salive, sueur ou haleine) dépend de plusieurs facteurs : facilité d'obtenir l'échantillon en toutes circonstances, disponibilité et fiabilité de tests rapides, corrélation avec les concentrations sanguines.

Pour les urines, il n'est pas toujours facile d'obtenir un échantillon au bord de la route en respectant l'intimité de la personne. Par contre, il existe plusieurs tests rapides fiables (voir plus loin). Certaines drogues et plus particulièrement le cannabis, peuvent rester détectables pendant plusieurs jours voire des semaines après consommation alors que leurs effets négatifs sur la capacité à conduire un véhicule ne durent généralement que quelques heures au maximum. La présence de certaines substances dans les urines est donc seulement indicative du fait qu'une personne a consommé une drogue et certainement pas qu'elle est sous influence. Ce problème peut être partiellement surmonté si les conducteurs devant êtres soumis à un test urinaire sont d'abord sélectionnés sur la base de signes physiques extérieurs, mais le test comportemental peut prendre bien du temps (45 - 60 minutes). Dans l'étude ROSITA, il a été démontré que si un test urinaire est réalisé après sélection des conducteurs suspects d'être sous influence des drogues, l' analyse urinaire (par CG-SM) est bien corrélée à la présence ou à l'absence de drogues dans le sang dans $94 \%$ des cas pour les amphétamines, $89 \%$ pour les benzodiazépines, $86 \%$ pour le cannabis, $97 \%$ pour la cocaïne et $86 \%$ pour les opiacés (7).

La salive est relativement facile à prélever chez les personnes qui ne sont pas sous influence de drogues, mais chez les consommateurs de cannabis, d'amphétamines ou de cocaïne, c'est beaucoup plus long (jusqu'à 15 minutes) et on obtient souvent un liquide très visqueux qui doit être prétraité avant de pouvoir être utilisé pour le test rapide. Il existe de nombreux prototypes de tests rapides, mais aucun n'est suffisamment fiable pour être utilisé en routine. Les nouveaux tests n'ont pas encore été évalués par des chercheurs indépendants. Dans une population présélectionnée par un test comportemental, la corrélation avec le résultat sanguin est comparable ou légèrement meilleure qu'avec les urines (7). Généralement, un résultat positif issu d'un test de salive peut être interprété avec un degré de certitude élevé comme résultant d'une consommation récente de produit, dans les 12, maximum 24 heures précédant le pré- lèvement, et non comme témoin d'une consommation ayant eu lieu plusieurs jours ou semaines avant le test. C'est surtout la molécule mère qui est présente, beaucoup plus que les métabolites. Il est donc essentiel que les immunoessais utilisent des anticorps spécifiques aux drogues et non à leurs métabolites.

La sueur (ou plutôt le dépôt sec qui reste sur la peau une fois que la sueur s'est évaporée) est très facile à prélever. Par contre, il n'existe qu'un test rapide, et il n'est pas validé pour le cannabis ; pour le THC, des concentrations très faibles devront être détectées. Vu la possibilité d'une contamination externe sur la peau ainsi que l'apparition plus tardive des drogues dans la sueur par comparaison au sang, la sueur semble moins appropriée que la salive pour détecter une consommation de drogue récente, et on peut s'interroger sur la pertinence de l'analyse de ce type de matrice lors de contrôles routiers. La drogue mère est facilement détectable dans les échantillons prélevés sur le front des conducteurs. La corrélation avec les résultats sanguins varie entre 78 et $97 \%$.(7). La compréhension du mécanisme du passage des drogues dans la sueur est loin d'être totale. Cependant, très récemment, Lester et coll. (8) ont étudié ce domaine plus en détail en ce qui concerne la cocaïne. La cocaïne semble se distribuer et disparaître rapidement de la surface de la peau de façon synchrone au plasma (8).

Un dépistage de drogue sur l'air expiré n'est pas pour demain. L'haleine est très facile à prélever, mais il n'existe aucun test rapide, et il y a eu très peu de recherches sur la durée de détection des drogues ou d'autres marqueurs dans l'air expiré.

Pour illustrer les résultats obtenus avec différents échantillons, nous donnons l'exemple du MDMA.

Dans une étude contrôlée sur les effets aigus d'une dose de MDMA sur les performances psychomotrices et cognitives, la concentration de MDMA a été déterminée dans le plasma, la salive, la sueur et l'urine pendant cinq heures consécutives, après l'administration d'une dose orale de $75 \mathrm{mg}$ de MDMA. La concentration maximale dans le plasma (moyenne $178 \mu \mathrm{g} / \mathrm{l}$ ) a été observée 2 à 3 heures après administration. Une bonne corrélation a été observée pour les profils d'évolution moyenne de la concentration sanguine et salivaire de MDMA. Cependant, une plus grande variabilité intraet interindividuelle à été observée pour la salive, avec des concentrations salivaires qui dépassent généralement celles du plasma. Même 4 à 5 heures après ingestion, la concentration moyenne de MDMA dans la sueur n'est que de $25 \mathrm{ng} /$ collecteur, ce qui est nettement inférieur aux valeurs mesurées lors de contrôles routiers. La différence entre un échantillon de sueur prélevé sur un individu en milieu hospitalier (tempéra- 
ture ambiante basse, personne relaxée, peau propre) et un échantillon collecté sur un conducteur venant d'une sortie «en boîte» (transpiration importante, peau sale) et arrêté par la police (situation de stress) pourrait expliquer ces écarts. De surcroît, la possibilité d'une contamination externe ainsi qu'une consommation répétée hors du cadre d'une étude contrôlée ne peut être écartée.

Pour établir une relation entre la concentration de MDMA dans les différentes matrices et le niveau de performance, des analyses de corrélation intra-individuelles ont été effectuées (C. Lamers, communication personnelle). Les corrélations intra-individu entre les concentrations de MDMA dans les différents liquides physiologiques et le changement de fréquence cardiaque et de tension artérielle après consommation de MDMA sont hautement significatives $(\mathrm{p}<0.01)$ et presque similaires pour le plasma, l'urine, et la salive et dans une moindre mesure pour la sueur. Pour l'urine et le plasma, une corrélation signifiante est observée pour les variations de température corporelle. Les concentrations en MDMA dans les liquides physiologiques sont corrélées de manière significative mais variables avec l'amélioration des performances psychomotrices après consommation de MDMA.

Les données sur la sueur pendant l'étude ROSITA (7), et les conclusions de Lester et coll. (8) montrent que cette matrice peut avoir une place dans le dépistage rapide d'une conduite sous influence.

La corrélation entre les concentrations de MDMA dans l'urine et les paramètres physiologiques et les tests de performance après consommation de MDMA est plutôt surprenante. Contrairement au cannabis, à la cocaïne et à l'héroïne, pour le MDMA et l'amphétamine, la molé-' cule mère est l'analyte cible dans l'urine. Une nouvelle approche consiste dans la détection de la molécule mère dans les urines (THC au lieu de THC-COOH, cocaïne au lieu de benzoylecgonine) pour contrôler la consommation récente, et pour estimer la présence d'un effet pharmacologique et la présence d'une drogue dans le sang. Par contre, une étude contrôlée ne tient pas compte d'une consommation répétée et d'autres facteurs tel que la variabilité de l'osmolalité urinaire et du $\mathrm{pH}$ de l'urine. Dans des circonstances moins contrôlées chez les utilisateurs réels, qui prennent souvent différentes doses successives, et où la consommation de boissons diffère selon les sujets, la corrélation entre les concentrations urinaires et les paramètres physiologiques pourrait être moins bonne.

\section{Les tests rapides}

D'un point de vue analytique, les tests de dépistage de drogues doivent être suffisamment sensibles afin de fournir un résultat positif pour les conducteurs ayant consommé des drogues et pouvoir détecter toutes les drogues visées par la législation, telles que les drogues de synthèse optimisées comme le MDMA. Ces tests doiventêtre suffisamment spécifiques afin de fournir un résultat positif chez les conducteurs ayant pris une drogue altérant la conduite ou visée par la loi sans réagir à des substances proches ne provoquant pas d'altération de la conduite.

\section{Les tests urinaires}

Actuellement, les outils se rapprochant le plus des tests d'haleine sont des tests immunochimiques non instrumentaux. Un inventaire des tests urinaires existant en 1999 a été publié (9).

Le projet Européen ROSITA a permis de comparer 15 tests urinaires différents chez 2968 participants dans 8 pays. Pour chaque type de drogue, plusieurs tests d'urine ont répondu aux critères analytiques minimum, à savoir une sensibilité et une spécificité d'au moins $90 \%$ et une précision d'au moins $95 \%$ par référence aux analyses de confirmations effectuées par CG-SM. Les résultats des différents tests sont résumés dans le tableau I. Aucun de ces tests n'a montré une performance optimale pour toutes les catégories de drogues. Cependant, certains tests sont parfaitement acceptables et ont généralement été utilisés comme un bon outil de prédiction des concentrations sanguines dans une population pré-sélectionnée de conducteurs, ce qui est en accord avec les résultats issus de l'évaluation de la procédure belge; des drogues ont été trouvées dans le sang de $85 \%$ des cas positifs aux tests psychomoteurs et urinaires (10). Contrairement à la police belge, plusieurs forces de police (surtout du nord de l'Europe) considèrent le recueil d'échantillons urinaires lors de contrôles routiers comme inacceptable. Des moyens appropriés (véhicules sanitaires) doivent être disponibles pour assurer l'intimité du donneur.

\section{Les tests rapides sur la salive}

$\mathrm{Vu}$ le caractère peu pratique et invasif du prélèvement d'un échantillon d'urine, depuis une dizaine d'années, le dépistage des drogues dans la salive est considéré par certains comme une sorte de panacée, qui permettrait de résoudre bien des problèmes. Dans l'étude ROSITA il a été démontré que la fiabilité des tests salivaires (Avitar, Cozart Rapiscan et Securetec Drugwipe) était insuffisante, avec beaucoup de faux positifs et faux négatifs (7).

De grands progrès ont été réalisés dans ce domaine, mais en ce début 2003, il n'y a pas encore de test salivaire fiable. A notre connaissance, il existe actuellement 8 tests rapides salivaires, dont plusieurs n'existent que sous forme de prototype. Les problèmes majeurs 


\begin{tabular}{|c|c|c|c|c|c|c|c|c|c|c|c|c|c|}
\hline \multirow{4}{*}{ 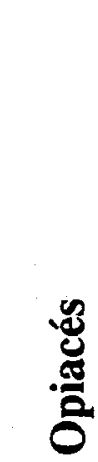 } & 甾 & $\approx$ & $\approx$ & $\infty$ & & a & à & \# & 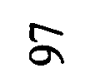 & ஃ & \& & ฉ̊ & 2 \\
\hline & की & $\approx$ & $n$ & 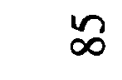 & & $\tilde{a}$ & g & $\sigma$ & $a$ & a & ஜ & ஜ & a \\
\hline & 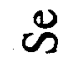 & $\stackrel{\infty}{\infty}$ & $\stackrel{\infty}{\circ}$ & 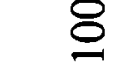 & & ' & $\stackrel{\infty}{\sim}$ & 5 & 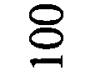 & $\infty$ & @ & $n$ & \& \\
\hline & $=$ & & $\stackrel{\infty}{\infty}$ & 商 & & $\bar{m}$ & 5 & $\underset{\sigma}{*}$ & 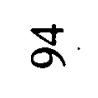 & $\stackrel{\infty}{\exists}$ & $\approx$ & $\stackrel{\infty}{\infty}$ & ஓ̊ \\
\hline \multirow{4}{*}{ 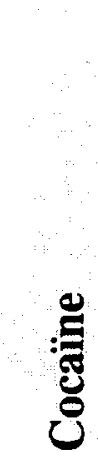 } & 爻 & $\propto$ & 5 & 2 & & \& & જ & 2 & 2 & 8 & ๙ & a & 8 \\
\hline & की & ळ & 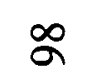 & 2 & & $\kappa$ & 8 & 2 & 2 & 8 & ๙ & 2 & 8 \\
\hline & 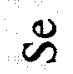 & 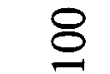 & $\infty$ & 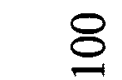 & & 8 & $\approx$ & $n$ & 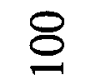 & \& & 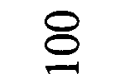 & 2 & $\approx$ \\
\hline & $=$ & 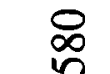 & $\tilde{\sigma}$ & $\stackrel{\infty}{\mathcal{I}}$ & & $\stackrel{2}{n}$ & 8 & $\stackrel{P}{n}$ & $\approx$ & $\cong$ & 8 & 苂 & ఃొ \\
\hline \multirow{4}{*}{ 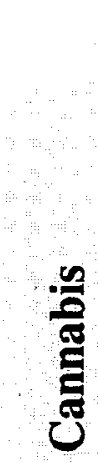 } & 甾 & $\alpha$ & $\approx$ & $\hat{a}$ & & Zू & $\infty$ & n & $\vec{a}$ & 8 & むু & ś & 8 \\
\hline & की & 8 & $\approx$ & $\sigma$ & & $\bar{a}$ & $\stackrel{\circ}{\circ}$ & 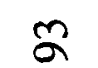 & 8 & 2 & $\alpha$ & 8 & ล \\
\hline & ஜ & 5 & $\alpha$ & \& & & á & $?$ & $\delta$ & $\infty$ & 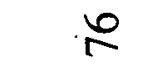 & à & $\sigma$ & 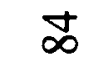 \\
\hline & $=$ & $\bar{n}$ & Oे & $\cong$ & & $\stackrel{\infty}{ \pm}$ & $\approx$ & 疋 & $\sigma$ & $\stackrel{\Xi}{\Xi}$ & $\infty$ & $\underset{\infty}{\infty}$ & \&్ల \\
\hline \multirow{4}{*}{ 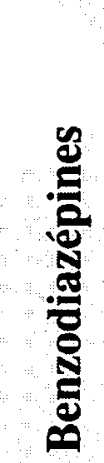 } & 甾 & a & $\infty$ & & & & $\vec{\sigma}$ & & & $\infty$ & & $\infty$ & ஃ \\
\hline & के & $\stackrel{\infty}{\circ}$ & $\Phi$ & & & & $\infty$ & & & $\infty$ & & ‡ & 2 \\
\hline & $\approx$ & $\bar{\sigma}$ & $\vec{\infty}$ & & & & 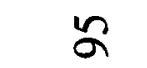 & & & வे & & œ & Z \\
\hline & $=$ & $\stackrel{\vec{N}}{\vec{N}}$ & $\stackrel{\mathscr{D}}{-}$ & & & & $\sigma$ & & & $\tilde{\Xi}$ & & 点 & 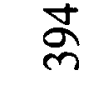 \\
\hline \multirow{5}{*}{ 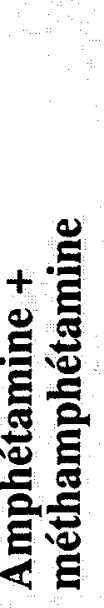 } & 病 & 2 & 8 & $\stackrel{\infty}{\circ}$ & $\infty$ & $\hat{a}$ & $\alpha$ & 2 & $\bar{\sigma}$ & \& & 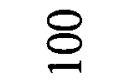 & 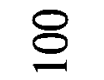 & $\approx$ \\
\hline & के & 2 & 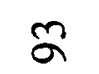 & @ & 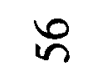 & 8 & ஜ & 8 & $\not$ & 2 & 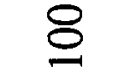 & 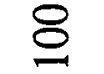 & 2 \\
\hline & $\ddot{~}$ & 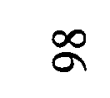 & $\hat{\infty}$ & sa & 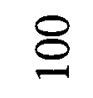 & $\infty$ & $\triangleright$ & $\approx$ & $\ddot{\infty}$ & $\sigma$ & \& & à & $\infty$ \\
\hline & $=$ & $\begin{array}{l}\infty \\
\& \\
q\end{array}$ & $\stackrel{\circ}{\infty}$ & I & $\infty$ & in & $\approx$ & స్ & $\sigma$ & \& & $\approx$ & $\stackrel{\infty}{n}$ & 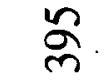 \\
\hline & & $\sum_{\text {至 }}$ & $\sum_{+}$ & $\sum_{+}$ & $\varangle$ & $\varangle$ & $\varangle$ & $\ll$ & $\varangle$ & $\sum_{+}$ & $\ll$ & $\sum_{+}$ & $\varangle$ \\
\hline & & 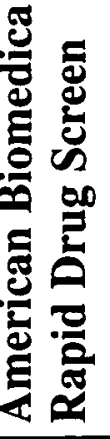 & 茔 & 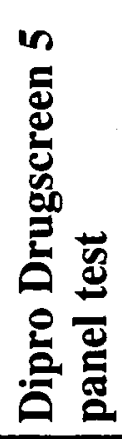 & 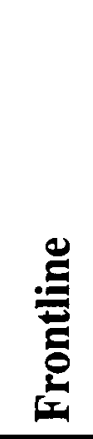 & 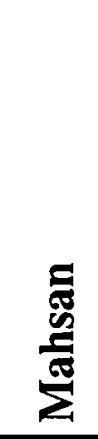 & 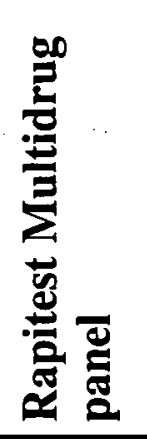 & 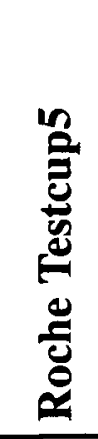 & 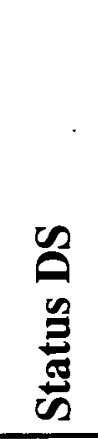 & 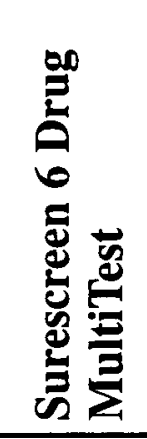 & 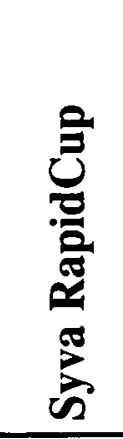 & 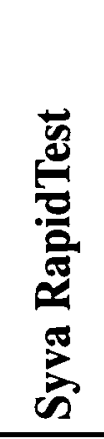 & 苋 \\
\hline
\end{tabular}


qui restent à résoudre sont la sensibilité pour le cannabis (à l'heure actuelle le test le plus sensible détecte $50 \mu \mathrm{g} / \mathrm{l}$ de tétrahydrocannabinol ; M. Walsh, communication personnelle) et les benzodiazépines, ainsi que le recueil et le transfert de l'échantillon chez les sujets qui ont peu de salive ou de la salive très visqueuse.

\section{Quels seuils pour les tests salivaires ?}

L'étude ROSITA a suggéré les seuils de positivité suivants: $1,9 \mu \mathrm{g} / \mathrm{l}$ pour le THC, $90 \mu \mathrm{g} / \mathrm{l}$ pour la somme des amphétamines et 5-10 $\mu \mathrm{g} / \mathrm{l}$ pour la cocaïne et les opiacés (7).

Les seuils de la SAMHSA pour les contrôles en milieu professionnel (tableau II) sont assez proches de ces seuils, et ils sont parfaitement utilisables dans des contrôles routiers. Dans une étude en Belgique, l'application des valeurs seuil fournies dans la version provisoire \#3 et la valeur seuil pour les amphétamines fournie dans la version \#4 a permis d'obtenir une valeur prédictive positive de $89-100 \%$ et une sensibilité de $91-100 \%$ pour le cannabis, les amphétamines et la cocaïne dans la salive collectée en crachant ; par référence aux résultats obtenus sur le plasma en accord avec les limites légales de confirmation. Le faible nombre d'échantillons positifs pour les opiacés n'a pas permis de formuler une conclusion finale (11). La présence de la drogue mère, à savoir la cocaïne et la 6MAM, dans la plupart des échantillons de salive des consommateurs de cocaïne et d'héroïne révèle une consommation récente de drogue.

Récemment, Kauert et coll. (12) ont présenté les résultats d'une étude comparative effectuée sur le sérum et la salive collectée sur 117 conducteurs suspectés de conduite sous influence. La salive a été collectée en utilisant un nouveau prototype de collecte et d'analyse nécessitant approximativement $200 \mu \mathrm{l}$ de salive pour confirmation : le Draeger Drug Test System ${ }^{\circledR}$. Les résultats issus de la comparaison entre les analyses effectuées par CG-SM sur la salive et le sérum sont en accord avec l'étude belge. La sensibilité est de $90 \%$ pour le cannabis et les amphétamines (MDMA inclus) et de $87 \%$ pour la cocaïne et l'héroïne. La spécificité et la précision pour la salive dépassent dans tous les cas les $90 \%$. De la cocaïne et de la 6-MAM ont été détectées dans approximativement $75 \%$ des échantillons de salive issus de sujets positifs pour la cocaïne et les opiacés. Les détails concernant les limites de quantification des méthodes de confirmation et la possible application de valeurs seuil n'ont pas été présentés (12).

Niedbala et al. ont mesuré les concentrations de THC dans des échantillons de salive recueillis avec le collecteur Intercept ${ }^{\circledR}$ après consommation de marijuana. Les concentrations baissent rapidement durant les premières $2-4 \mathrm{~h}$; cependant une variation substantielle est observée entre individus durant l'élimination. L'utilisation de valeurs seuil de dépistage de $1,5 \mu \mathrm{g} / \mathrm{l}$ et de valeurs seuil de confirmation de $0,5 \mu \mathrm{g} / \mathrm{l}$ de THC, a permis d'obtenir des résultats positifs consécutifs durant $13 \pm 3$ heures sur les échantillons de salive dilués. Une fréquence de détection substantiellement plus élevée d'échantillons de salive positifs a été observée durant les huit premières heures par rapport aux résultats issus de l'analyse des urines. Cette caractéristique unique est de la plus grande importance lorsque l'on tient compte de la courte période d'influence de la marijuana sur les performances. De plus, une bonne corrélation entre l'évolution des concentrations en THC dans le plasma et la salive a été suggérée (13).

Tableau II : Seuils proposés pour les drogues dans la salive.

\begin{tabular}{|l|l|c|c|}
\hline Classe de drogue & \multirow{2}{*}{$\begin{array}{l}\text { Analytes (*analyte } \\
\text { cible pour le dépistage) }\end{array}$} & \multicolumn{2}{|c|}{ Seuils $(\boldsymbol{\mu g} / \mathrm{l})$} \\
\cline { 3 - 4 } & Dépistage & Confirmation \\
\hline Amphétamines & d-méthamphétamine* & $160^{(1)} / 50^{(2)}$ & $160^{(1)} / 50^{(2)}$ \\
& d-amphétamine & & $160^{(1)} / 50^{(2)}$ \\
\hline Cocaïne & benzoylecgonine* & 20 & 8 \\
\hline Opiacés & morphine* & 40 & 40 \\
& 6-acétymorphine & & 4 \\
\hline Cannabis & $\Delta$-9-tetrahydrocannabinol* & 4 & $2^{(1)} / 4^{(2)}$ \\
\hline
\end{tabular}

(1) Substance Abuse and Mental Health Service Administration. Mandatory guidelines for federal workplace drug testing programs. Draf\#3, December 2000

${ }^{(2)}$ Substance Abuse and Mental Health Service Administration. Mandatory guidelines for federal workplace drug testing programs. Draft\#4, September 2001

\section{Technique de prélèvement}

Le succès du dépistage de la salive est certainement lié au protocole de recueil, assurant le prélèvement reproductible d'un volume adéquat de liquide pas trop visqueux. Une méthode standardisée de collecte est préconisée si des résultats quantitatifs doivent être produits. Un crachat contient des débris cellulaires, des particules de nourriture et des filets de mucus ; la grande viscosité de la salive complique l'échantillonnage et le test. Un échantillon de salive recueilli avec un outil adapté fournit généralement un échantillon plus propre. Un grand nombre d'outils de recueil est disponible.

Cependant, les études effectuées sur l'effet de la méthode de recueil sur les concentrations des drogues dans la salive sont rares. Les concentrations en codéine sont en moyenne 3,6 fois plus élevées dans un crachat obtenu sans stimulation, que dans la salive obtenue par stimulation acide et de 1,3 à 2 fois plus élevées que 
dans la salive collectée en mâchant une gomme sans sucre ou en utilisant deux outils de recueil spécifiques (14). Des concentrations substantiellement différentes en THC ont été obtenues en fonction de la méthode de recueil (crachat ou Salivette ${ }^{\circledR}$ ). Des rendements d'extraction insuffisants dus à des phénomènes d'adsorption sur le collecteur ont aussi été mis en évidence (11).

\section{Les tests rapides sur la sueur}

Le Drugwipe ${ }^{\circledR}$, qui était le seul outil de dépistage proposé pour la sueur, a seulement donné des résultats valables pour la détection de l'amphétamine et du MDMA, ceci en comparant les résultats du dépistage sur le terrain (en essuyant le front) et les résultats de la confirmation par CG-SM sur la sueur et le plasma.

\section{Les test rapides sur l'air expiré}

Depuis les années 70 , certains chercheurs ont essayé de détecter les drogues dans l'air expiré. Les techniques analytiques utilisées à cette époque n'étaient pas très sensibles et/ou étaient sujetes à d'autres problèmes (par exemple la contamination des étalons internes deutérés par des composés non deutérés), et ces recherches n'ont pas été couronnées de succès (15).

\section{Détection d'une conduite sous influence par le labo- ratoire ?}

Avec une législation analytique ou per se, la présence de drogues dans le sang (au-dessus d'un certain seuil) correspond à une conduite sous influence. Avant de passer en revue les techniques analytiques existantes, quelques questions concernant les seuils seront abordés.

\section{Seuils de danger ou seuils analytiques?}

Dans tous les pays qui ont introduit une législation analytique, il y a eu des discussions sur les seuils à utiliser. Comme pour l'alcool, on demande à partir de quelle concentration sanguine, le risque d'accident est augmenté. Malheureusement, il y a très peu de données de type Grand Rapids pour les drogues. Drummer a obtenu des données sur les concentrations sanguines de THC chez plus de 4000 conducteurs australiens impliqués dans un accident mortel dans le contexte d'une étude de responsabilité. Les résultats ont confirmé que la probabilité d'être responsable pour l'accident est plus élevée dans le groupe de conducteurs où l'analyse sanguine montrait une consommation de drogue que dans le groupe où ni drogue ni alcool n'étaient détéctés. Le risque d'être considéré coupable d'un accident mortel avait augmenté d'un facteur 2,8 pour les conducteurs positifs au THC, et dans les cas où les concentrations sanguines de THC étaient $\geq 5 \mu \mathrm{g} / 1$ l'augmentation du risque était d'un facteur 7,0 (16).

La mauvaise corrélation entre la concentration sanguine de par exemple le THC et les effets (hystérésis avec les effets avant le pic plasmatique et diminution rapide des effets alors que la concentration sanguine de THC a déjà fortement diminué) nous laisse supposer que ces seuils de danger seront très difficiles sinon impossibles à déterminer. De plus, choisir des seuils de danger équivaut à tolérer l'usage de drogues, ce qui reste illégal dans presque tous les pays. En effet, si on met un seuil de danger dans la loi, on aura très rapidement des questions comme: est-ce que je peux fumer un demi joint sans être positif lors d'un contrôle routier?

En l'absence de ces seuils de danger, mais aussi pour les raisons de principe expliquées ci-dessus, tous les gouvernements ont opté pour des seuils analytiques.

\section{Quels seuils analytiques utiliser?}

Comme on peut le voir dans le tableau III, il n'y a pas de consensus sur le seuil analytique entre les différents pays. Si l'on part de la définition, un seuil analytique est la concentration minimale que les laboratoires peuvent détecter de manière fiable. En Allemagne, les seuils sont déterminés par la «Grenzwertkommission», qui les a récemment revus à la baisse. En Suède, les seuils analytiques sont les limites de détection du laboratoire national. En Belgique, les seuils légaux ont été basés sur les recommandations de la SFTA. Une harmonisation est souhaitable, mais elle est rendue difficile par les différents types d'échantillons utilisés (sérum en Allemagne, plasma en Belgique, sang total en France et en Suède), et par les conséquences différentes d'un résultat positif : sanction administrative en Allemagne, sanction pénale en Belgique. Nous pensons que les seuils seront progressivement abaissés comme pour l'alcool.

\section{Méthodes}

Pour le dosage des drogues dans le sang, la SFTA a recommandé différentes méthodes par chromatographie en phase gazeuse, couplée à la spectrométrie de masse, qui sont largement utilisées (17-19). Il existe également quelques revues exhaustives très récentes à ce sujet, comme par exemple celle de Möller et Kraemer (20), qui font le point depuis une revue antérieure (21). La CG-SM reste la méthode la plus utilisée pour les analyses de confirmation des échantillons de sérum, plasma ou sang total. Par contre, des méthodes de chromatographie en phase liquide couplée à la spectrometrie de masse (CL-SM) ont aussi été introduites 
Tableau III : Seuils analytiques ( $\mu g /$, sauf Suède : $n g / g$ ) des différentes drogues dans quatre pays européens.

\begin{tabular}{|l|c|c|c|c|c|}
\hline \multirow{2}{*}{} & \multicolumn{2}{|c|}{ Allemagne } & Belgique & France & Suède \\
\cline { 2 - 6 } & 1998 & $\begin{array}{c}2002 \\
(29)\end{array}$ & & & \\
\hline Amphétamine & 50 & 25 & 50 & 50 & 30 \\
\hline MDMA & 50 & 25 & 50 & 50 & 20 \\
\hline MDEA & 50 & 25 & 50 & 50 & 20 \\
\hline MDA & & & & 50 & 20 \\
\hline Cocaïne & & & 50 & 50 & 20 \\
\hline Benzoylecgonine & 150 & 75 & 50 & 50 & 20 \\
\hline Morphine (libre) & 20 & 10 & 20 & 20 & 5 \\
\hline THC & 2 & 1 & 2 & 1 & 0.3 \\
\hline
\end{tabular}

pour les différents classes de drogues pour la confirmation ou le dépistage et la confirmation en une seule étape $(22,23)$.

Au cours des dernières années, des progrès ont été faits dans le domaine des méthodes immunologiques pour le dépistage des stupéfiants dans le sang. Une adaptation de la méthode CEDIA (Cloned enzyme donor immunoassay) a été publiée (24). Les résultats étaient satisfaisants pour la plupart des tests, à l'exception des amphétamines où la sensibilité était insuffisante. Certains laboratoires font d'abord un dépistage par immunoessai ELISA (enzyme-linked immunoassay) avant de confirmer les positifs. Différents auteurs ont validé ces tests (25-27). L'expérience montre qu'il y a des interférences dans les échantillons prélevés sur fluorure de sodium/oxalate de potassium.

Pour la salive, plusieurs laboratoires disposent maintenant d'une méthode par chromatographie liquide couplée à la spectrométrie de masse en tandem, qui permet de doser les drogues principales sur un échantillon de $250 \mu \mathrm{L}$. La méthode de Mortier (28) permet le dosage sensible des amphétamines, opiacés et cocaïne avec une limite de détection de $2 \mu \mathrm{g} / \mathrm{l}$ sur un échantillon de $200 \mu \mathrm{L}$, après extraction par une méthode en phase solide. La méthode de Wood (23), limitée aux amphétamines, ne dure que 20 minutes et a une limite de détection de $2 \mu \mathrm{g} / \mathrm{l}$.

\section{Contrôle de qualité}

Différents contrôles de qualité sont disponibles pour le dosage des drogues dans le sang ou le plasma. La SFTA organise un contrôle chaque année et la GTFCh allemande organise trois contrôles sur 3 échantillons chaque année. Des contrôles commerciaux sont disponibles chez Medichem (Steinenbronn, Allemagne).

\section{Conclusion}

Depuis le dernier numéro spécial de Toxicorama consacré aux drogues et conduite automobile, publié en 1996, il n'y a pas eu beaucoup d'évolution dans les méthodes d'analyses des drogues dans le sang par CGSM. La CL-SM-SM semble présenter des avantages pour certaines drogues, mais elle n'est pas encore utilisée couramment. La CL-SM-SM pourrait devenir la méthode de choix pour la confirmation des drogues dans la salive. Il n'y a pas de consensus en Europe sur les seuils analytiques, qui continueront à faire l'objet de beaucoup de discussions.

La place des tests rapides de terrain est mieux définie. Les besoins des services de police sont clairement établis, mais pour les tests salivaires il reste des problèmes non résolus, comme la sensibilité pour le cannabis et la difficulté d'analyser les échantillons très visqueux. En l'absence de tests salivaires fiables, certains pays utilisent les tests urinaires sans rencontrer de grandes difficultés.

\section{Références}

1. Borkenstein R.F. The role of the drinking driver in traffic accidents (the Grand Rapids Study). Second edition. Blutalkohol $1974 ; 11$ Suppl.1

2. Krüger H.P., Perrine M.W. Bud, Mettke M., Huessy F.B. Overview of the legal provisions, difficulties faced by police, and analysis of prevention attempts in selected European countries. In: Road Traffic and Drugs (ISBN 92-871-4145-2). Strasbourg: Council of Europe publishing, $2000 ; 87-185$.

3. Möller M.R., Steinmeyer S., Aberl F. Deliverable D3: Operational, user and legal requirements across EU member states for roadside drug testing equipment. In: ROSITA, Roadside Testing Assessment. Ghent University, $2001 ; 103-66$.

4. Verstraete A.G. Deliverable D5: General conclusions and recommendations. In: ROSITA, Roadside Testing Assessment. Ghent University, 2001 ; 393-97.

5. Verstraete A.G., Maes V.A. The elaboration of the per se legislation on drugs and driving in Belgium. Problems of Forensic Sciences (Z Zagadnie Nauk Sadowych) 2000 ; XLII : 189-94.

6. Steinmeyer S., Ohr H., Maurer H.J. Möller M.R. Practical aspects of roadside tests for administrative offences in Germany. Forensic. Sci. Int. 2001 ; 121 : 33-6.

7. Verstraete A.G., Puddu M. Deliverable D4: Evaluation of different roadside drug tests. In: ROSITA, Roadside Testing Assessment. Ghent University, 2001 ; 167-232.

8. Lester L., Uemura N., Ademola J., Harkey M.R., Nath R.P., Kim S.J., Jerschow E., Henderson G.L., Mendelson J., Jones R.T. Disposition of cocaine in skin, interstitial fluid, sebum, and stratum corneum. J. Anal. Toxicol. $2002 ; 26$ : 547-53. 
9. Samyn N., Viaene B., Vandevenne L., Verstraete A. Inventory of state-of-the-art roadside drug testing equipment. In: ROSITA, Roadside Testing Assessment. Ghent University, 2001 ; 45-102.

10. Maes V., Samyn N., Willekens M., De Boeck G., Verstraete A. Stupéfiants et conduite automobile - les actions réalisées en Belgique. Ann. Toxicol. Anal. 2003 ; 15(2) : 128-37.

11. Samyn N., De Boeck G., Verstraete A. The use of oral fluid and sweat wipes for the detection of drugs of abuse in drivers. J. Forensic Sci. $2002 ; 47: 1380-7$.

12. Kauert G., Moeller M., Maurer H., Steinmeyer S., Toennes S.W. Statistical evaluation of analytical findings from corresponding blood and oral fluid taken at the roadside. In : Marquet P., ed. Proceedings of the 40th TIAFT meeting. Paris, August 2002, in press.

13. Niedbala R.S., Kardos K.W., Fritch D.F., Kardos S., Fries T., Waga J. et al. Detection of Marijuana use by oral fluid and urine analysis following single-dose administration of smoked and oral marijuana. J. Anal. Toxicol. 2001 ; $25: 289-303$.

14. O'Neal C.L., Crouch D.J., Rollins D.E., Fatah A.A. The effects of collection methods on oral fluid codeine concentrations. J. Anal. Toxicol. $2000 ; 24$ : 536-42.

15. Verstraete A.G. Perspectives for the detection of cannabis in breath. In: Proceedings of the 15th International conference on alcohol, drugs and traffic safety (CD-rom). Stockholm, May 2000.

16. Drummer O.H., Chu M., Gerostamoulos J. The forensic toxicology of (9-tetrahydro-cannabinol (THC). In : Marquet P., ed. Proceedings of the 40th TIAFT meeting. Paris, August 2002, in press.

17. Gaillard Y., Pepin G., Marquet P., Kintz P., Deveaux M., Mura P. Identification et dosage de la benzoylecgonine, cocaine, méthylecgonine-ester, codéine, morphine et 6acétylmorphine dans le sang total. Toxicorama $1996 ; 8$ : 17-22.

18. Marquet P., Lachâtre G., Kintz P., Pepin G., Deveaux M., Mura P. Identification et dosage des principales drogues amphétaminiques dans le sang total par chromatographie en phase gazeuse couplée à la spectrométrie de masse (CPG-SM). Toxicorama $1996 ; 8: 23-8$.

19. Kintz P., Cirimele V., Pepin G., Marquet P., Deveaux M., Mura P. Identification et dosage des cannabinoides dans le sang total. Toxicorama $1996 ; 8: 29-33$.

20. Möller M.R., Kraemer T. Drugs of abuse monitoring in blood for control of driving under the influence of drugs. Ther. Drug Monit. 2002 ; 24 : 210-21.

21. Möller M.R., Steinmeyer S., Kraemer T. Determination of drugs of abuse in blood. J. Chromatogr. B. 1998 ; $713: 91-110$

22. Marquet P. Progress in liquid chromatography-mass spectrometry in clinical and forensic toxicology. Ther. Drug Monit. 2002 ; 24 : 255-76.

23. Wood M., De Boeck G., Samyn N., Morris M., Cooper D.P., Maes R.A.A., De Bruijn E.A. Development of a rapid and sensitive method for the quantitation of amphetamines in human plasma and oral fluid by LC-MS-MS. J. Anal. Toxicol. $2003 ; 27: 78-87$.
24. Iwersen B.S., Schmoldt A. Direct semi-quantitative screening of drugs of abuse in blood by means of CEDIA DAU immunoassays. J. Anal. Toxicol. 1999 ; 23 : 247-56.

25. Kemp P., Sneed G., Kupiec T., Spiehler V. Validation of microtiter plate ELISA for screening of postmortem blood for opiates and benzodiazepines. J. Anal. Toxicol. $2002 ; 26: 504-12$.

26. Kupiec T., DeCicco L., Spiehler V., Sneed G., Kemp ·P. Choice of an ELISA assay for screening postmortem blood for amphetamine and/or methamphetamine. J. Anal. Toxicol. $2002 ; 26: 513-18$.

27. Moore K.A., Werner C., Zannelli R.M., Levine B., Smith M.L. Screening postmortem blood and tissues for nine classes of drugs of abuse using automated microplate immunoassay. Forensic Sci. Int. 1999 ; 106 : 93-102.

28. Mortier K.A., Maudens K.E., Lambert W.E., Clauwaert K.M., Van Boxlaer J.F., Deforce D.L., Van Peteghem C.H., De Leenheer A.P. Simultaneous, quantitative determination of opiates, amphetamines, cocaine and benzoylecgonine in oral fluid by liquid chromatography quadrupole-time-of-flight mass spectrometry J. Chromatogr. B $2002 ; 779: 321-30$.

29. Grenzwertkommission: Beschluss zu $\S 24 a$ (2) StVG vom 20.11.2002. Toxichem + Krimtech $2002 ; 69: 127$. 